\title{
Spatiotemporal distribution of highly pathogenic avian influenza subtype H5N8 in Korea
}

\author{
Woo-Hyun Kim${ }^{1}$, Sun Hak Bae ${ }^{2}$, and Seongbeom Cho ${ }^{1}$ \\ ${ }^{1}$ Seoul National University \\ ${ }^{2}$ Kangwon National University
}

November 25, 2020

\begin{abstract}
In zoonotic disease, highly pathogenic avian influenza (HPAI) is a major threat to human and poultry health worldwide. In January 2014, HPAI virus subtype H5N8 first infected poultry farms in South Korea, and a total of 393 outbreaks were reported with enormous economic damage in the poultry industry. We analyzed the spatiotemporal distribution of HPAI H5N8 outbreaks in poultry farms using the global and local spatiotemporal interactions in the first outbreak wave from January 2014 to June 2015 and the second wave from September 2014 to June 2015. The space-time K-function analyses revealed significant interactions within three days and over $40 \mathrm{~km}$ in two study periods in global spatiotemporal interaction. The excess risk attributable value (D0) was maintained despite the distance in the case of HPAI H5N8 in Korea. Eleven spatiotemporal clusters were identified, and the results show the HPAI introduction from the southwestern region and the spread to the middle region in Korea. Six clusters were distributed in 0.46-9.86 km space and 3-19 days in time, while five clusters were distributed in 19.74-72.59 km space and 27-36 days in time. This global and local spatiotemporal interaction indicates that the HPAI epidemic in Korea was mostly characterized by short period transmission within a small area and dispersed by long-range jumps. This finding supports strict control strategies such as preemptive depopulation, the standstill, and poultry movement tracking. More studies are needed to understand HPAI disease transmission patterns of HPAI in Korea.
\end{abstract}

\section{Hosted file}

Manuscript_spatio_temporal_com.pdf available at https://authorea.com/users/378563/articles/ 495032-spatiotemporal-distribution-of-highly-pathogenic-avian-influenza-subtype-h5n8-inkorea 


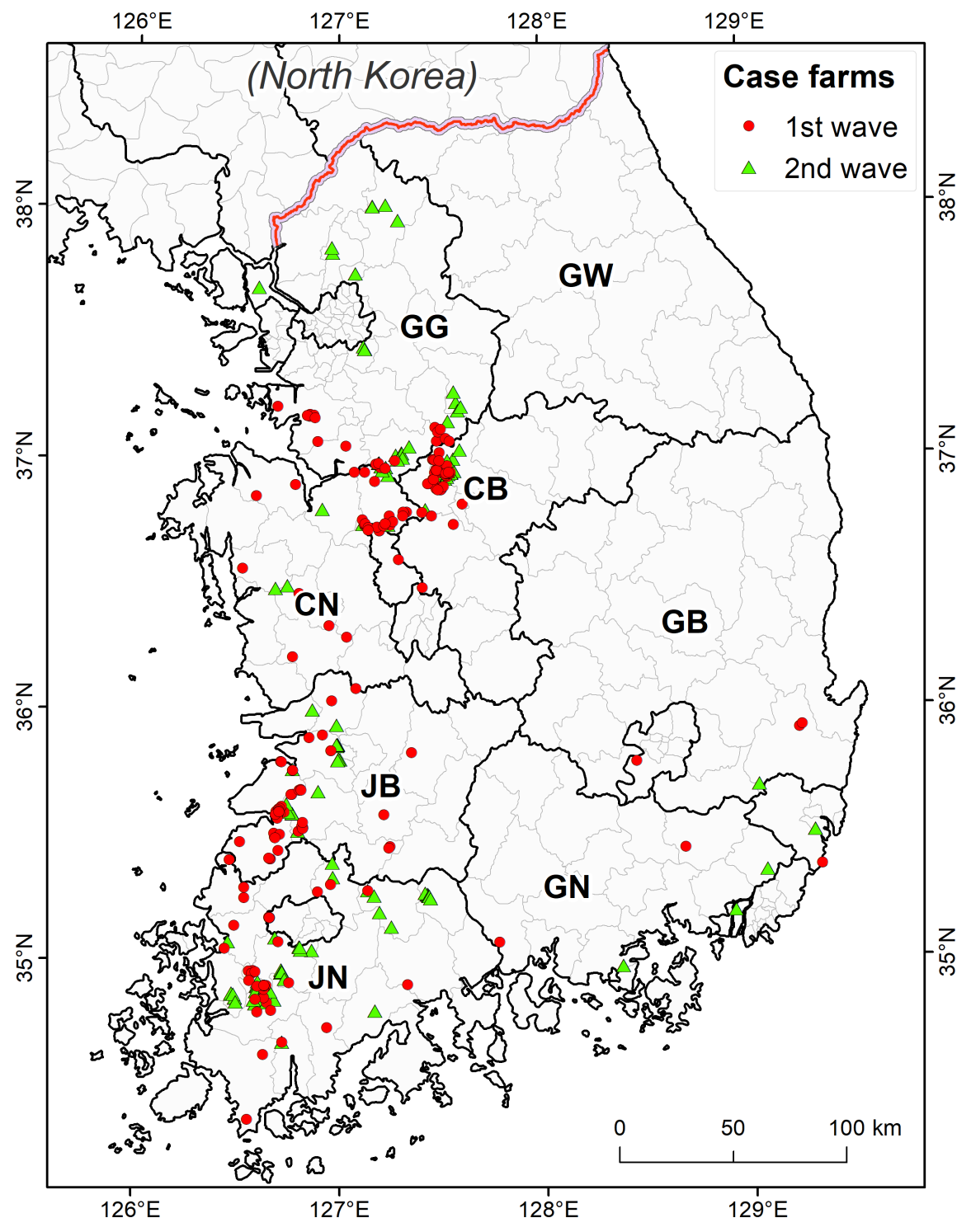



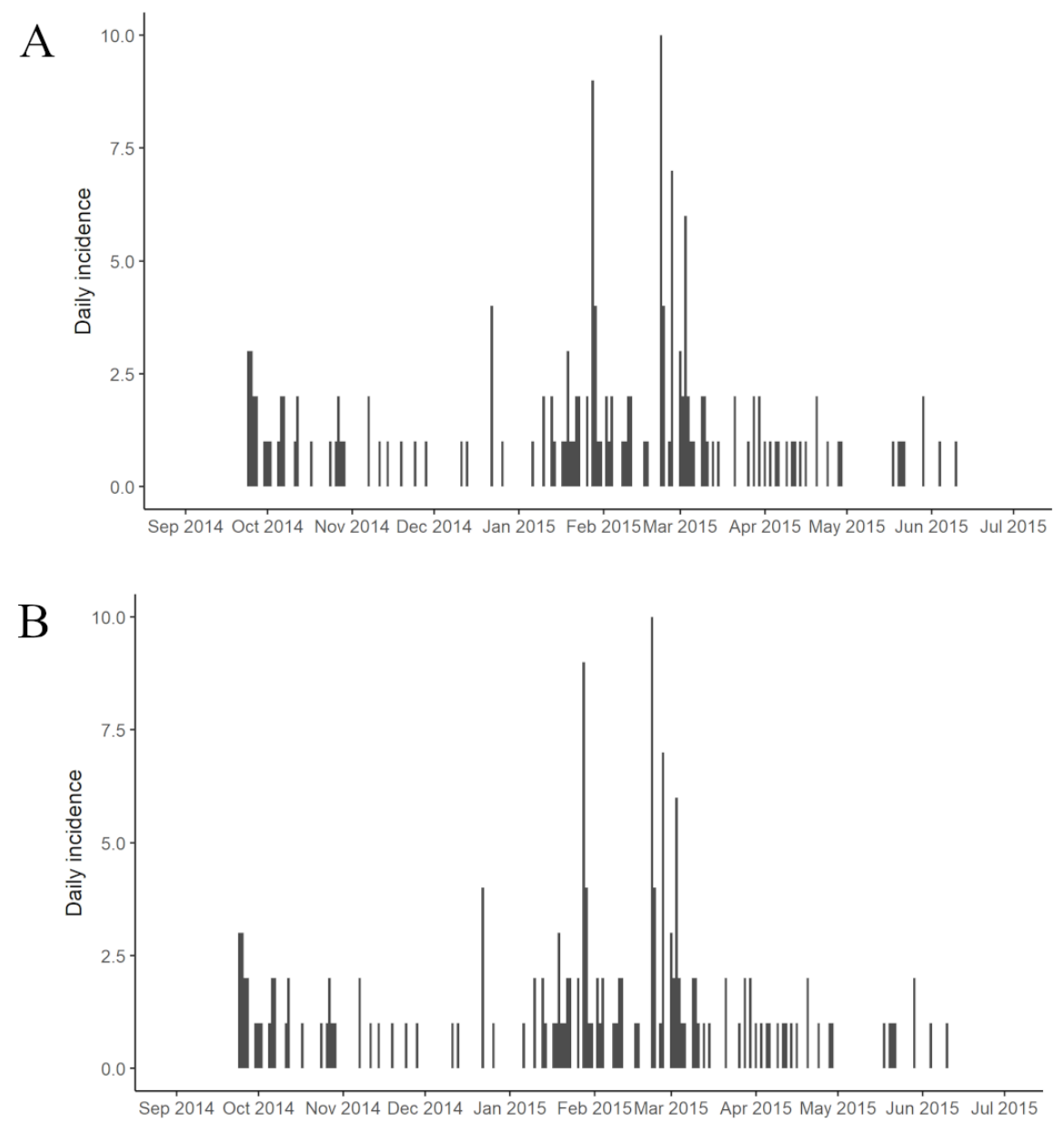

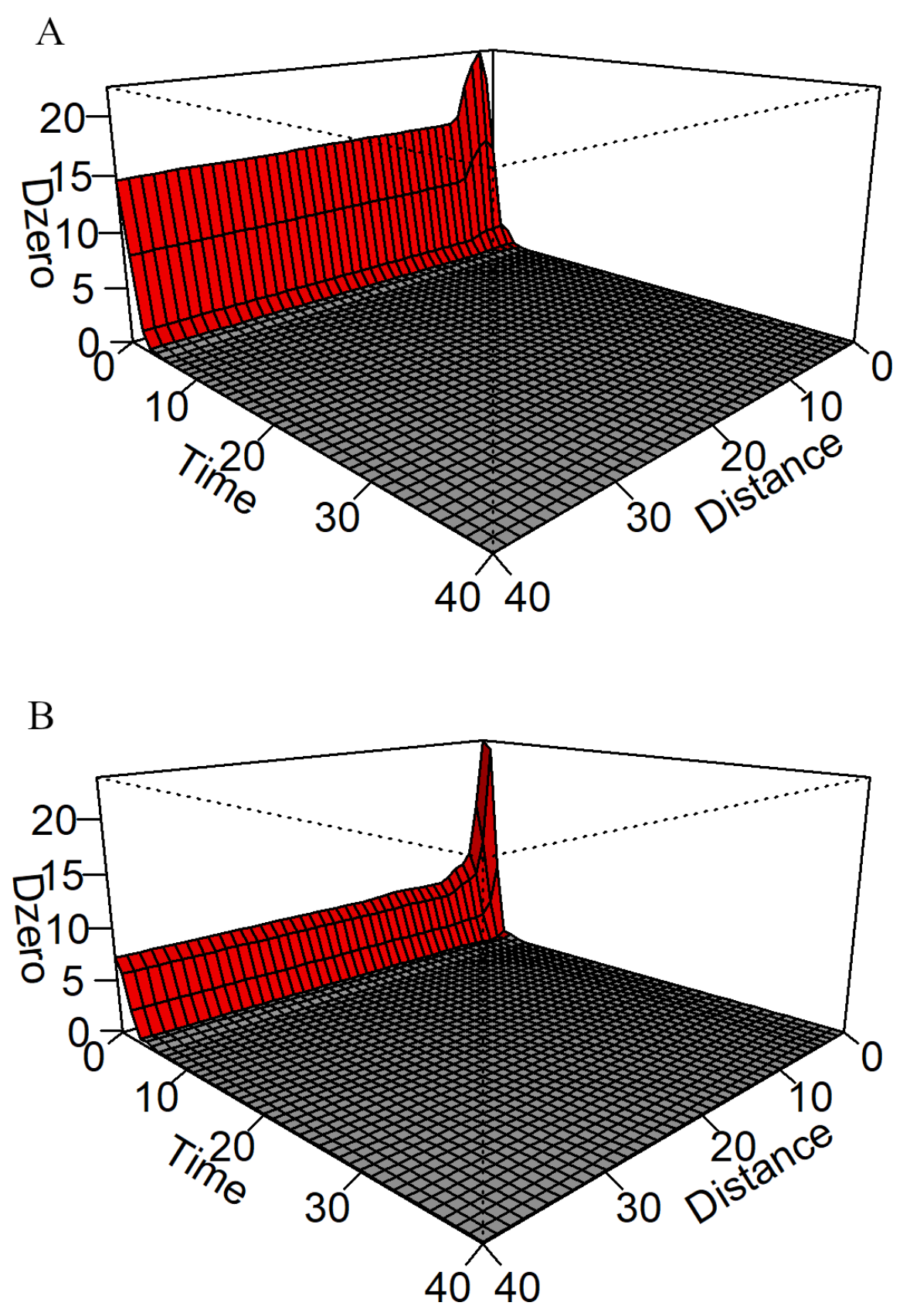

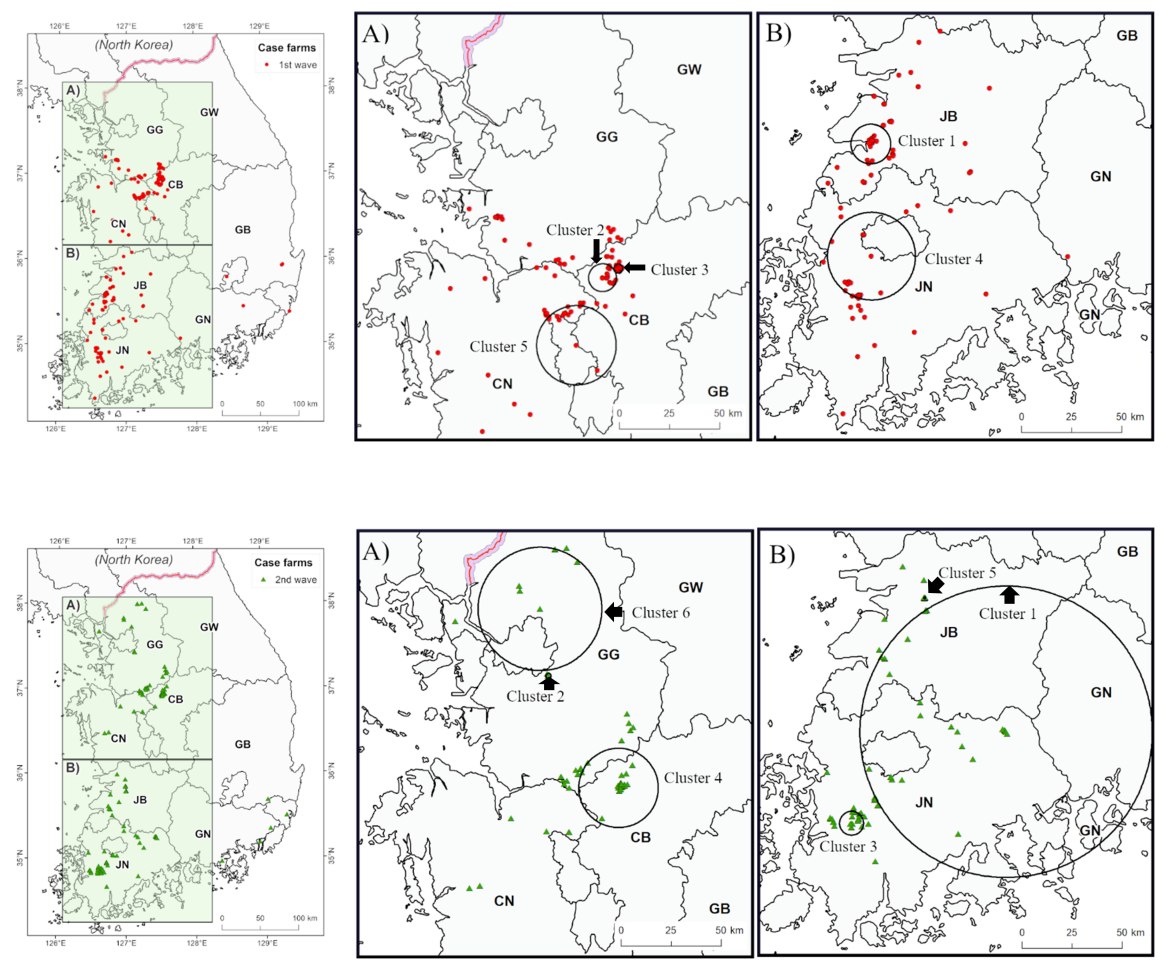